\title{
Convergence of metrics under self - dual Weyl tensor and Scalar curvature bounds
}

\author{
Yiyan $\mathrm{Xu}$
}

November 21, 2018

\begin{abstract}
We establish a $C^{1, \alpha}$ compactness theorem for the metrics with bounded self - dual Weyl tensor and Scalar curvature. The key step is to estimate the $C^{1, \alpha}$ harmonic radius, where we use the blow up analysis as in Anderson90. The result is motivated by, and may be applied to the Calabi flow on complex surfaces Calabi82.
\end{abstract}

\section{Introduction}

In Riemannian Geometry, it is interesting to consider the convergence and finiteness in the presence of basic geometric data. With the fundamental Cheeger - Gromov convergence theory, this problem is reduced to the harmonic radius estimate in a standard way. In a remarkable piece of work, Jost and Karcher obtained a explicit estimate on the harmonic radius which depends only on lower volume, upper diameter and sectional curvature bound Jost84 GW88. The most important feature about harmonic coordinates is that the metric is apparently controlled by the Ricci curvature, roughly speaking, to a determined system of P.D.E., whereas assuming bounds on $R \mathrm{~m}$ corresponds to a overdetermined system information. This was exploited by Anderson90, i.e. the bounds $\mid$ Ric $\mid \leq \lambda$ and $i n j \geq i_{0}$ alone imply a lower bound on the harmonic radius without any assumptions on the curvature tensor.

Without the lower bound on the injectivity radius but relaxes to a lower bound on volume, there are examples which exhibit that the degeneration may occur. Pursuing this phenomena of thought, geometers had made a well known conjecture: the convergence is $C^{1, \alpha}$ off a singular set of Hausdorff codimension at least four CCT02.

Key steps in Andersen's proof are to establish the continuous properties of the harmonic radius, regard as a function, under the $C^{k, \alpha}(k \geq 1)$ or $W^{k, p}(k \geq 1)$ convergence, which in turn ultimately depends on some type of partial differential equations estimate. Besides the elliptic regularity theory, the main ingredient in the Andersen's results is a blow up argument that relates the validity of convergence/rigidity theorems to the global behavior of complete, non - compact manifolds with critical metric. The hypothesis on the manifolds, such as 
curvature bound and lower bound on injectivity radius, was basically only used in the characterization of the Euclidean space, i.e. rigidity theorem. It is likely that some weakening of the hypothesis, but still valid in the rigidity theorem, suffices to give a lower bound on the harmonic radius.

We proceed with another generalization of the metrics with bounded Ricci curvature to scalar curvature bound, but at the expense of some hypotheses on the structure of metric, for example, Anti - self - dual or Kähler metric. The motivation here also comes from the Calabi flow. In estimating whether the solution of Calabi flow exists and convergence, one crucial estimate which still lacking is one showing that the space of all kähler metrics $\omega$ in a fixed cohomoplogy class $\Omega$, and for which the scalar curvature $|S(\omega)|_{\omega}$ is bounded, is compact in the $C^{1, \alpha}$ Cheeger - Gromov topology, see Calabi82 and ChHe08.

Theorem 1.1. Let $M^{4}$ be a compact oriented four dimensional manifold, let $\left\{g_{i}\right\}$ be a sequence metrics on $M^{4}$ with

1. either bounded self - dual Weyl tensor, i.e., $\left|W^{+}\left(g_{i}\right)\right| \leq \Lambda$ or $\left(M, g_{i}\right)$ is Kähler metric;

2. bounded scalar curvature, i.e., $\left|S\left(g_{i}\right)\right| \leq \Lambda$;

3. unit volume, i.e., $\left|\operatorname{Vol}\left(M_{i}, g_{i}\right)\right| \equiv 1$;

4. bounded Sobolev constant $C_{S}\left(g_{i}\right) \leq C_{S}$, i.e.,

$$
\left\{\int_{M}|v|^{4} d \nu_{g_{i}}\right\}^{\frac{1}{2}} \leq C_{S} \int_{M}|d v|_{g_{i}}^{2} d \nu_{g_{i}}, \forall v \in C_{0}^{0,1}(M) .
$$

Then there exist a subsequence $\{j\} \subset\{i\}$ such that $\left(M, g_{j}\right)$ converge to a compact multi - fold $\left(M_{\infty}, g_{\infty}\right)$ in the Gromov - Hausdorff topology. Moreover, on the regular set $M_{\infty} \backslash\left\{x_{1}, \cdots, x_{m}\right\}$, the metric $g_{\infty}$ is $C^{1, \alpha}$ and the convergence is in the $C^{1, \alpha}$ Cheeger - Gromov topology; while each of the singular point $x_{i}$ have a neighborhood homeomorphic to finite disjoint union of cones $C\left(S^{3} / \Gamma\right)$ with identifications of vertex, where $\Gamma$ is finite subgroup of $O(4)$.

Remark 1. By the Gauss - Bonnet formula and the Hirzebruch signature formula Besse87, then we have

$$
\int_{M^{4}}|R m|^{2}=-24 \pi^{2} \tau(M)-8 \pi^{2} \chi(M)+\int_{M} \frac{1}{12} S^{2}+4\left|W^{+}\right|^{2}
$$

If the scalar curvature $S$ and self - dual Weyl tensor has uniformly bounded $L^{2}$ norm, then we have a prior $L^{2}-$ bound on curvature tensor $R m$. The above theorem holds, with out fixing topology, under the additional assumption that

$$
\int_{M_{i}^{4}}\left|R m\left(g_{i}\right)\right|^{2} \leq \Lambda_{1}
$$


Remark 2. In fact, under the controlled topology, i.e. $b_{1}(M) \leq b_{0}$, the full Sobolev constant bound can be released to a lower volume growth assumption, i.e. $\operatorname{Vol}(B(x, r)) \geq V_{0} r^{n}$. Gang Tian and Jeff Viaclovsky's proof for Critical metric [TV08] also holds in our case, since the volume is continuous in the $C^{1, \alpha}$ - topology.

Analogous convergence result also hold for the metric with bounded Bach tensor and $C^{2}$ bound of the Scalar curvature, which is actually easier to prove since we have the precise elliptic system (3.10).

Theorem 1.2. Let $\left\{M_{i}, g_{i}\right\}$ be a sequence of Riemannian manifolds with

1. either bounded Bach tensor, i.e., $\left|B\left(g_{i}\right)\right| \leq \Lambda$ or $\left\{M_{i}, g_{i}\right\}$ is Kähler metric;

2. $C^{2}$ bounded scalar curvature, i.e., $\left|\nabla^{2} S\left(g_{i}\right)\right| \leq \Lambda$;

3. unit volume, i.e., $\left|\operatorname{Vol}\left(M_{i}, g_{i}\right)\right| \equiv 1$;

4. bounded Sobolev constant $C_{S}$ in (1.1);

5 . bounded $L^{\frac{n}{2}}-$ norm of curvature, i.e.,

$$
\left\{\int_{M}|R m|^{\frac{n}{2}}\right\}^{\frac{n}{2}} \leq \Lambda_{1} .
$$

Then there exist a subsequence $\{j\} \subset\{i\}$ such that $\left(M_{j}, g_{j}\right)$ converge to a compact multi - fold $\left(M_{\infty}, g_{\infty}\right)$ in the $C^{3, \alpha}$ topology off finite singularity (as theorem 1.1).

Example 1. (Schoen87])There exists a family of conformally flat metrics with constant scalar curvature $g_{t}$ on $S^{1} \times S^{3}(t \geq 1)$ such that the diameter of $S^{1} \times\{x\}$ goes to 0 as $t \rightarrow \infty$ for a point $x \in S^{3}$. For other point $y \neq x$ the diameter of the slice $S^{1} \times\{y\}$ is bounded from below. The limit space is the quotient space of $S^{4}$ which identifies the north pole and the south pole.

Example 2. ([KT87] Nakajima92) Let $M_{\infty}$ be an orbifold given by the $\mathbb{Z} / 2 \mathbb{Z}$ - quotient of the complex 2- torus $T^{2}=\mathbb{C}^{2} / \mathbb{Z}^{4}$, where $-1 \in \mathbb{Z} / 2 \mathbb{Z}$ acts on $T$ by

$$
\left(z_{1}, z_{2}\right) \bmod \mathbb{Z}^{4} \mapsto\left(-z_{1},-z_{2}\right) \bmod \mathbb{Z}^{4} .
$$

The flat metric on $T$ descends to an orbifold metric $g_{\infty}$ on $M_{\infty}$, and it is an orbifold Ricci flat anti - self dual metric. Moreover, $M_{\infty}$ has a complex manifold structure (with singularities) since the $\mathbb{Z} / 2 \mathbb{Z}$ - action is holomorphic. Let us take the minimal resolution $\pi: M \rightarrow M_{\infty}$. The singularities are sixteen simple singularities of type $A_{1}$. The minimal resolution $M$ is called a Kummer surface and is an example of K3 surfaces. Let $\mathcal{S}=\left\{x_{1} \cdots, x_{16}\right\}$ be the singular set, and let $E_{1}, \cdots, E_{16}$ be the exceptional divisor. These are complex submanifolds of $M$ biholomorphic to $\mathbb{C} P^{1}$ with the self - intersection number -2 . By the solution of the Calabi conjecture we have a unique Calabi - Yau metric in each Kähler class, which is automatically anti - self - dual. Take a Kähler class, 
and there exist a sequence of Ricci - flat Kahler metric $g_{i}$, as follows: (1) the volume of $M$ with respect to $g_{i}$ is equal to $1 ;(2)$ the volume of the exceptional divisor $E_{k}$ is equal to $\frac{1}{i}$ for $k=1, \cdots, 16$. It can be shown that the metric $g_{i}$ converges to $\pi^{*} g_{\infty}$ over $M \backslash \cup_{k=1}^{16} E_{k}$, but condition (2) forces the metric to become degenerate along $E_{k}$ as $i \rightarrow \infty$, i.e. $E_{k}$ collapse to a point, and the Riemannian curvature concentrates along $\cup_{k=1}^{16} E_{k}$. Moreover, the curvature concentrates so completely that the limit metric is a flat orbifold - metric.

Acknowledgements: The author would like to thank his advisor Professor Gang Tian for suggesting this problem and constant encouragement. The author would also like to thank Yalong Shi for numerous suggestions which helped to improve the presentation.

\section{Self - Dual Weyl Curvature}

Riemannian geometry in dimension 4 has some special features which are not present in any other dimension. In dimensions $n \geq 5$ the group $S O(n)$ is simple, and the space of Weyl tensors is irreducible under $S O(n)$, whereas in dimension 4 we have $S O(4)=S O(3) \times \times_{\mathbb{Z}_{2}} S O(3)$, and a decomposition of the space of Weyl tensors into two $S O(4)$ - irreducible components. The fact that $S O(4)$ is not simple is reflected at the Lie algebra level in the the decomposition of the bundle of 2 forms into self - dual and anti - self - dual parts under the Hodge star operator.

Let $\left(M^{4}, g\right)$ be a oriented four dimensional Riemannian manifold. The Hodge star operator $*$ associated to $g$ takes $\Lambda:=\Lambda^{2} T M$ to itself and satisfying $* *=1$,

$$
\varphi \wedge * \psi=\langle\varphi, \psi\rangle d v o l_{g}
$$

where $\langle\cdot, \cdot\rangle$ denotes the inner product on $\Lambda$ induced by $g$. Then $\Lambda$ admits a decomposition of the form

$$
\Lambda=\Lambda^{+} \oplus \Lambda^{-},
$$

where $\Lambda^{ \pm}$is the \pm 1 eigenspace of $*$. The curvature operator $R m$, viewed as an endomorphism on $\Lambda$, has the following matrix expression Besse87:

$$
R m=\left[\begin{array}{cc}
W^{+}+\frac{S}{12} \operatorname{Id}_{\Lambda^{+}} & \stackrel{\circ}{\text { Ric }} \\
(\text { Ric })^{t} & W^{-}+\frac{S}{12} \operatorname{Id}_{\Lambda^{-}}
\end{array}\right]
$$

where $\stackrel{\circ}{\text { Ric }} \in \operatorname{Hom}\left(\Lambda^{+}, \Lambda^{-}\right)$is the trace free part of the Ricci curvature, $S$ is the scalar curvature, and $W^{ \pm} \in S_{0}^{2}\left(\Lambda^{ \pm}\right)$is the (anti -) self - dual part of the Weyl tensor, $S_{0}^{2}$ denote traceless symmetric endomorphisms. If we denote the projection operator by

$$
P_{ \pm}:=\frac{1}{2}(1 \pm *): \Lambda \rightarrow \Lambda^{ \pm}
$$

then

$$
W^{ \pm}=P_{ \pm} \circ R m \circ P_{ \pm}-\frac{S}{12} \operatorname{Id}_{\Lambda^{ \pm}}
$$


Definition 2.1. Let $\left(M^{4}, g\right)$ be a compact Riemannian four dimensional Riemannian manifold, the metric $g$ is called anti - self - dual, if $W^{+}=0(\Leftrightarrow * W=$ $-W)$.

We note that reversing the orientation transfers the self - dual part to the anti - self - dual part. For anti - self - dual metric, if we reverse the orientation, then $W^{-}(g)=0$ and $g$ is called to be self dual $\left(W^{-}\left(\mathbb{C} P^{2}, J, \omega_{F S}\right)=0\right.$, i.e. $\left(\overline{\mathbb{C} P^{2}}, J, \omega_{F S}\right)$ is anti - self - dual).

There are many interesting examples of anti - self - dual metrics. First, the (anti -) self - duality of the metric is a conformally invariant property. In particular, the conformal flat metrics will be anti - self - dual. Second, a large number of anti - self - dual metrics are Kähler metrics with zero scalar, since the self - dual part of the Weyl tensor is given by Besse87. (Proposition 16.62)

$$
W^{+}=\operatorname{diag}\left(\frac{S}{6},-\frac{S}{12},-\frac{S}{12}\right)
$$

In particular, a K3 surface with Calabi - Yau metric is anti - self - dual. This also follows from Gauss - Bonnet formula and the Hirzebruch signature formula, since with the canonical orientation $\tau=-16$ and $\chi=24$.

Definition 2.2. (Bach tensor) Let $\left(M^{n}, g\right)$ be a compact Riemannian manifold, the the Bach tensor is defined by

$$
B_{i j}=\frac{1}{n-3} \nabla^{k} \nabla^{l} W_{i k j l}+\frac{1}{n-2} R^{k l} W_{i k j l}
$$

Such Bach tensor is symmetric, trace free and divergence free 2 tensor. In dimension 4, Bach tensor arise as the Euler - Lagrange equations of the functional on the $L^{2}$ - norm of the Weyl curvature tensor. Using the Bianchi identities we may rewrite the Bach flat (Kähler) metric with constant scalar curvature as an elliptic system TV05a,

$$
\left\{\begin{array}{l}
\Delta R m=L\left(\nabla^{2} R i c\right)+R m * R m \\
\Delta R i c=R m * R i c
\end{array}\right.
$$

In dimension 4, using Bianchi identity, Bach tensor can also be written as $B_{i j}=2 \nabla^{k} \nabla^{l} W_{i k j l}^{+}+R^{k l} W_{i k j l}^{+}$, thus anti - self - dual metric will be Bach flat. As we have mentioned earlier, the Kähler metric on complex surface with zero scalar curvature will be anti - self -dual, so it is also Bach flat. For higher dimension, Kähler with constant scalar metric will still satisfies the equation (2.1).

Let $M$ be a closed oriented smooth manifold. A smooth Riemannian metric $g$ on $M$ is a smooth section of the bundle $S^{2} T^{*} M$ of positive definite symmetric 2 - tensors. The space $\mathcal{M}$ of all Riemannian metrics on $M$ is a convex open cone in $\Gamma\left(S^{2} T^{*} M\right)$. A Riemannian metric is given locally by functions, so we can define the (Sobolev) norm on $\mathcal{M}$ with respect to some fixed metric, and the prescribed curvature condition can be viewed as partial differential equation on $\mathcal{M}$. 
With this viewpoint, we want to use a priori estimate of Elliptic equation to study the convergence theory for the metric with bounded self - dual Weyl tensor. Note that self - dual Weyl tensor $W^{+}(g)$ is equivariant under the action of the diffeomorphism transformation and conformal change, but if we fix the gauge, the prescribe self - dual Weyl tensor does form an elliptic system. In fact, it is now a standard technique when we studying the geometry problems, such as compactness of geometric Calculus of Variations(Yang - Mills instanton, Einstein metric), DeTurck trick for existence theory of geometric (Ricci) flow...... For anti - self dual structure, it is well known that the local structure of moduli spaces is controlled by an elliptic deformation complex KK92 Tian08. For reader's convenience, we would like make it more clear in a PDE view point (2.2). As a consequence, we get a crucial estimates for this paper, i.e. a priori $L^{p}$ estimate (2.5) for the metric with bounded self - dual Weyl tensor and Scalar curvature.

Theorem 2.3. Let $M$ be a closed oriented smooth 4- manifold, we consider the following map (equation):

$$
\begin{aligned}
L: \mathcal{M} & \longrightarrow S_{0}^{2} \Lambda^{+} \oplus C^{\infty}(M) \oplus T M \\
g & \longmapsto\left(W^{+}(g), S(g), \tau_{g, \bar{g}}(i d)\right)
\end{aligned}
$$

where $\tau_{\tau_{g, \bar{g}}}(i d):=\operatorname{tr}_{g} \nabla^{g \otimes \bar{g}} d(i d)$ is the tension field of the identity map id : $(M, g) \longrightarrow(M, \bar{g})$, and $\bar{g}$ is some fixed background metric.

Then the principle symbol $\sigma(L)$ of the linearized operator of $L$ at $(x, g)$ is injective, which is given as follows: for any $\xi \in T_{x} M$ and $h \in S_{x}^{2} T M$,

$$
\sigma(L)(x, \xi)(h)=\left(h_{\Lambda^{+}},-\operatorname{tr}(h)|\xi|^{2}+h(\xi, \xi), h(\xi, \cdot)-\frac{1}{2} \operatorname{tr} h \cdot \xi\right),
$$

where $h_{\Lambda^{+}}$is defined by (2.4).

In particular, the equation (2.2) on metric is an elliptic system of partial differential equations of mixed order. And consequently, the $L^{p}$ theory will holds:

$$
\|g\|_{W^{2, p}} \leq C\left(\|g\|_{L^{p}}+\left\|W^{+}(g)\right\|_{L^{p}}+\|S(g)\|_{L^{p}}+\left\|\tau_{g, \bar{g}}\right\|_{W^{1, p}}\right) .
$$

Proof. Recall the self - dual part of Weyl tensor $W^{+}$is defined by

$$
W^{+}=P_{+} \circ R m \circ P_{+}-\frac{S}{12} \operatorname{Id}_{\Lambda^{+}},
$$

then

$$
\begin{aligned}
\delta W_{g}^{+}(h)= & P_{+} \circ \delta R m_{g}(h) \circ P_{+}+\delta P_{+g}(h) \circ R m \circ P_{+}+P_{+} \circ R m \circ \delta P_{+g}(h) \\
& -\frac{1}{12} \delta S_{g}(h) \operatorname{Id}_{\Lambda^{+}}-\frac{S}{12} \delta \operatorname{Id}_{\Lambda_{g}^{+}}(h) \\
= & P_{+} \circ \delta R m_{g}(h) \circ P_{+}-\frac{1}{12} \delta S_{g}(h) \operatorname{Id}_{\Lambda^{+}}+\text {l.o.t. }
\end{aligned}
$$


On the other hand, the first variation of curvature is Besse87:

$$
\begin{aligned}
\delta R m_{g}(h) & (X, Y, Z, U)=\frac{1}{2}[h(R(X, Y) Z, U)-h(R(X, Y) U, Z)] \\
& +\frac{1}{2}\left[\nabla_{Y, Z}^{2} h(X, U)+\nabla_{X, U}^{2} h(Y, Z)-\nabla_{X, Z}^{2} h(Y, U)-\nabla_{Y, U}^{2} h(X, Z)\right]
\end{aligned}
$$

Note that the scalar term $\delta S_{g}(h) \operatorname{Id}_{\Lambda^{+}}$does not contribute to the the principle symbol of the second operator of $\delta W_{g}^{+}$, since $\delta W_{g}^{+}$is traceless. Therefore, by taking the traceless symmetric part, the principle symbol of the second operator of $\delta W_{g}^{+}$is given by

$$
\begin{aligned}
\sigma\left(\delta W_{g}^{+}\right)(x, \xi)(h)= & \sigma\left(P_{+} \circ \delta R m_{g} \circ P_{+}\right)(x, \xi)(h) \\
& -\frac{1}{3} \operatorname{tr}_{\Lambda^{+}}\left(\sigma\left(P_{+} \circ \delta R m_{g} \circ P_{+}\right)(x, \xi)(h)\right) g_{\Lambda^{+}} \\
= & h_{\Lambda^{+}}
\end{aligned}
$$

where $h_{\Lambda^{+}}$is defined as follows: for $e, e^{\prime} \in \Lambda^{+}(x)$, and $\left\{e_{i}\right\}$ is an orthonormal basis of $\Lambda^{+}(x)$, we have

$$
h_{\Lambda^{+}}\left(e, e^{\prime}\right)=h\left(e(\xi), e^{\prime}(\xi)\right)-\frac{1}{3} \sum_{i=1}^{3} h\left(e_{i}(\xi), e_{i}(\xi)\right) g_{x}\left(e, e^{\prime}\right) .
$$

The liberalization of the scalar curvature map is given by

$$
\delta S_{g}(h)=\Delta \operatorname{tr} h+\delta^{2} h-g(\text { Ric }, h),
$$

then its symbol is

$$
\sigma\left(\delta S_{g}\right)(x, \xi)(h)=-g(\xi, \xi) \operatorname{tr} h+h(\xi, \xi) .
$$

The liberalization of the the tension field map is given by

$$
\delta \tau_{g, \bar{g}}(i d)(h)=\langle\nabla d(i d), h\rangle-\left\langle\delta h+\frac{1}{2} d(\operatorname{tr} h), d(i d)\right\rangle,
$$

therefore,

$$
\sigma\left(\delta \tau_{g, \bar{g}(i d)} g\right)(x, \xi)(h)=h(\xi, \cdot)-\frac{1}{2} \operatorname{tr} h \xi .
$$

Combining the above three symbol computation gives (2.3).

Now we will verify that the symbol of $\sigma(L)$ is injective, i.e. $\forall \xi \neq 0$, $\sigma(L)(x, \xi)(h)=0$ implies that $h=0$. It is easy to check that $\sigma(L)(x, \xi)(h)=0$ implies $h_{\Lambda^{+}}=0, h(\xi, \xi)=0, \operatorname{tr} h=0$.

First choose an orthonormal basis $X_{1}, X_{2}, X_{3}, X_{4}$ for $T_{x} M$, then

$e_{1}=\frac{\sqrt{2}}{2}\left(X_{1} \wedge X_{2}+X_{3} \wedge X_{4}\right), e_{2}=\frac{\sqrt{2}}{2}\left(X_{1} \wedge X_{3}+X_{4} \wedge X_{2}\right), e_{3}=\frac{\sqrt{2}}{2}\left(X_{1} \wedge X_{4}+X_{2} \wedge X_{3}\right)$ 
gives an orthonormal bases of $\Lambda_{x}^{+}$. For any $\xi=\xi^{i} X_{i}$ with $|\xi|=1$, we have

$$
\left[\begin{array}{c}
e_{1}(\xi) \\
e_{2}(\xi) \\
e_{3}(\xi) \\
\frac{\sqrt{2}}{2} \xi
\end{array}\right]=\frac{\sqrt{2}}{2}\left[\begin{array}{cccc}
\xi^{2} & -\xi^{1} & \xi^{4} & -\xi^{3} \\
\xi^{3} & -\xi^{4} & -\xi^{1} & \xi^{2} \\
\xi^{4} & \xi^{3} & -\xi^{2} & -\xi^{1} \\
\xi^{1} & \xi^{2} & \xi^{3} & \xi^{4}
\end{array}\right]\left[\begin{array}{c}
X_{1} \\
X_{2} \\
X_{3} \\
X_{4}
\end{array}\right]:=\frac{\sqrt{2}}{2} U X
$$

If $h_{\Lambda^{+}}=0$ and $h(\xi, \xi)=0$, from (2.4), then it is equivalent to

$$
\left[\begin{array}{cc}
\left(h\left(e_{i}(\xi), e_{j}(\xi)\right)\right. & 0 \\
0 & \frac{1}{2} h(\xi, \xi)
\end{array}\right]=\frac{1}{2} U h U^{T}=\frac{1}{3} \sum_{i} h\left(e_{i}(\xi), e_{i}(\xi)\left[\begin{array}{ll}
I & 0 \\
0 & 0
\end{array}\right]\right.
$$

Since $U \in O(4)$, then we have

$$
h=\frac{2}{3} \sum_{i} h\left(e_{i}(\xi), e_{i}(\xi) U^{T}\left[\begin{array}{cc}
I & 0 \\
0 & 0
\end{array}\right] U\right.
$$

Furthermore, if $\operatorname{tr} h=0$, then

$$
0=\operatorname{tr} h=2 \sum_{i} h\left(e_{i}(\xi), e_{i}(\xi),\right.
$$

and consequently, $h=0$.

The symbol $\sigma(L)$ is injective, by comparing the dimension ( $\operatorname{dim}=10)$, we conclude that the equation (2.2) is an elliptic system of partial differential equations with mixed order. For an elliptic system (of mixed order), we have a priori estimate, for example, the Schauder or $L^{p}$ theory [Morrey66. Alternatively, if we replace the tension field $\tau_{g, \bar{g}}(i d)$ in equation (2.2) by $\mathcal{L}_{\tau_{g, \bar{g}}(i d)} g$, then it will be a overdetermined elliptic system of second order partial differential equations. In fact, the a priori estimate ( $L^{p}$ theory) hold for differential operators between vector bundles is equivalent to the injectivity of the symbol, while the 'solubility criteria' holds if the symbol is surjective (theorem 19.25 in Palais68 or theorem A.8 in [DK90]).

In local harmonic coordinates, since the metric is $C^{1, \alpha}$ close to the Euclidean metric, if necessary, we can make the harmonic norm $C$ small enough, then the operator $L$ is uniformly elliptic with $C^{1, \alpha}$ continuous leading terms, by the $L^{p}$ theory, we have the estimate

$$
\begin{aligned}
\|g\|_{W^{2, p}\left(B_{r}\right)} & \leq C\left(\|g\|_{L^{p}\left(B_{2 r}\right)}+\left\|W^{+}(g), S(g)\right\|_{L^{p}\left(B_{2 r}\right)}+\left\|\tau_{g, \bar{g}}(i d)\right\|_{W^{1, p}\left(B_{2 r}\right)}\right) \\
& =C\left(\|g\|_{L^{p}\left(B_{2 r}\right)}+\left\|W^{+}(g)\right\|_{L^{p}\left(B_{2 r}\right)}+\|S(g)\|_{L^{p}\left(B_{2 r}\right)}\right)
\end{aligned}
$$

The last step holds, since $\tau_{\tau_{g, \bar{g}}}(i d)=0$ if we identify the geodesic ball with the Euclidean ball under harmonic coordinate.

\section{Convergence Theory}

To generalize the convergence theory with assumption on sectional curvature to weaker curvature bounded hypothesis, it is convenient to use the concept of 
harmonic radius, which was introduced and developed in Anderson90 AnCh92 Petersen97.

Definition 3.1. (Harmonic Radius) Let $(M, g)$ be a Riemannian manifold, fixing any $m \in \mathbb{N}$, given any $x \in M$, there is $r=r(x)$, for which we can find a harmonic coordinate system

$$
\left(\left\{x_{i}\right\}_{i}^{n}\right): B(x, r) \subset(M, g) \rightarrow \mathbb{R}^{n}
$$

such that

1. the coordinate function $\left(\left\{x_{i}\right\}_{i}^{n}\right): B(x, r) \subset(M, g) \rightarrow \mathbb{R}^{n}$ is harmonic;

2. the metric tensor $g_{i j}:=g\left(\nabla x_{i}, \nabla x_{j}\right)$ is $C^{m, \alpha}$ bounded on $B(x, r)$, i.e.

$$
e^{-C} \delta_{i j} \leq g_{i j} \leq e^{C} \delta_{i j} \text { (as bilinear forms), }
$$

and

$$
\sum_{1 \leq|\beta| \leq m} r^{|\beta|} \sup \left|\partial^{\beta} g_{i j}\right|+\sum_{|\beta|=m} r^{m+\alpha}\left[\partial^{\beta} g_{i j}\right]_{\alpha} \leq C,
$$

for some fixed constant $C \geq 0$, where the norms are taken with respect to the coordinates $\left(\left\{x_{i}\right\}_{i}^{n}\right)$ on $B(x, r)$. We say that $x \in(M, g)$ admits a harmonic coordinate with bounded $C^{m, \alpha}-$ norm on the scale $r$ :

$$
\|x \in(M, g)\|_{C^{m, \alpha}, r} \leq C .
$$

Moreover, we let $r_{h}(x)$ be the $C^{m, \alpha}$ harmonic radius at $x$, which is defined as the radius of the largest geodesic ball about $x$, on which there are $C^{m, \alpha}$ harmonic coordinates, i.e.

$$
r_{h}(x)=\sup \left\{r>0 \mid\|x \in(M, g)\|_{C^{m, \alpha}, r} \leq C\right\} .
$$

The $C^{m, \alpha}$ harmonic radius of $(M, g)$ is defined by $r_{h}(M)=\inf _{x \in M} r_{h}(x)$.

Definition 3.2. For a compact metric space $X$, define the covering number of the geodesic ball on the scale $\epsilon$ as follows

$$
\operatorname{Cov}(\epsilon)=\min \left\{n \mid \exists\left\{x_{i}\right\}_{i=1}^{n} \subset X, \bigcup_{i=1}^{n} B\left(x_{i}, \epsilon\right)=X, B\left(x_{i}, \frac{\epsilon}{2}\right) \bigcap_{i \neq j} B\left(x_{j}, \frac{\epsilon}{2}\right)=\emptyset\right\}
$$

Now, let us state the fundamental theorem of convergence theory:

Theorem 3.3. (Anderson90 Petersen97) For given $n \geq 2, C \geq 0, N>0$, $\alpha \in(0,1]$ and $r_{0}>0$, consider the class $\mathcal{M}\left(n, C, N, r_{0}\right)$ of $n$ - dimensional Riemannian manifolds

$$
\left\{(M, g) \mid r_{h}(x) \geq r_{0},\|x \in(M, g)\|_{C^{m, \alpha}, r_{0}} \leq C, \forall x \text { and } \operatorname{Cov}\left(\frac{r_{0}}{10}\right) \leq N .\right\}
$$

Then $\mathcal{M}\left(n, C, N, r_{0}\right)$ is compact in the $C^{m, \beta}$ Cheeger - Gromov topology for all $\beta<\alpha$. Moreover, the theorem also valid for bounded domains in Riemannian manifolds, as well as for pointed complete Riemannian manifolds, provided one works on compact subsets. 
Theorem 3.4. (TV05a TV08 Carron10) Let $(M, g)$ be a complete Riemannian manifold or Riemannian multi - fold with finite point singularities, $g$ is critical metric (for example, Bach flat with zero scalar curvature). Assume that $\left(M^{n}, g\right)$ satisfies the Sobolev inequality,

$$
\left\{\int_{M}|v|^{\frac{2 n}{n-2}} d \nu\right\}^{\frac{n-2}{n}} \leq C_{S} \int_{M}|d v|^{2} d \nu, \forall v \in C_{0}^{0,1}(M) ;
$$

and the curvature has bounded $L^{\frac{n}{2}}$ norm,

$$
\left\{\int_{M}|R m|^{\frac{n}{2}} d \nu\right\}^{\frac{n}{2}} \leq \epsilon
$$

Then $\left(M^{n}, g\right)$ has finite many ends, which is ALE of order two. Moreover, the volume is at most Euclidean volume growth, $\operatorname{Vol}(B(p, r)) \leq V r^{n}$ for some positive constant $V=V\left(n, C_{S}, \epsilon\right)$.

Moreover, if $(M, g)$ is smooth and $\epsilon=\epsilon_{0}$ is small enough, which depends on $n$ and the Sobolev constant $C_{S}$, then $(M, g)$ is isometric to the Euclidean space $\left(\mathbb{R}^{n}, g_{E}\right)$.

Lemma 3.5. For any $m \geq 2$, let $B(r):=B\left(x_{0}, r\right)$ be a geodesic ball in a compact oriented four Riemannian manifold $\left(M^{4}, g\right)$, where $g$ has bounded self - dual Weyl tensor (or Kähler) and bounded scalar curvature, $\left|W^{+}\right|+|S| \leq \Lambda$. Then there exist a positive constant $\epsilon_{0}=\epsilon\left(C_{S}\right)$ and $\kappa_{0}=\kappa_{0}\left(C_{S}, \Lambda\right)$ such that if

$$
\left\{\int_{B(x, 2 r)}|R m|^{2} d \nu\right\}^{\frac{1}{2}} \leq \epsilon_{0},
$$

then for all $x \in B\left(x_{0}, r\right)$, the $C^{1, \alpha}$ harmonic radius $r_{h}(x)$ satisfies

$$
\frac{r_{h}(x)}{\operatorname{dist}(x, \partial B)} \geq \kappa_{0}>0 \text {. }
$$

Proof. On a smooth fixed smooth Riemannian manifold $(M, g)$, it is clear that the harmonic radius $r_{h}(x)$ is positive or saying (3.2) holds, but $\kappa_{0}$ depends on $(M, g)$ and $x$. Thus, we must show that $\kappa_{0}$ depends only on the hypothesis prescribed in the lemma.

We argue by contradiction, which is similar as the blow up analysis for Ricci curvature case in Anderson90. If (3.2) is false, then there are sequence of Riemannian 4 manifolds $\left\{\left(M_{i}, g_{i}\right)\right\}$ with the bounds in the lemma, and points $x_{i} \in B_{i}(r) \subset\left(M_{i}, g_{i}\right)$ such that

$$
\frac{r_{h}\left(x_{i}\right)}{\operatorname{dist}\left(x_{i}, \partial B_{i}\right)} \rightarrow 0, \quad \text { as } i \rightarrow \infty .
$$

We may assume, with out lose of generality, that the points $x_{i}$ realize the minimum of the left side of (3.2) and

$$
\left\|x_{i} \in\left(M_{i}, g_{i}\right)\right\|_{C^{1, \alpha}, r_{h}\left(x_{i}\right)} \in\left[\frac{C}{2}, C\right] .
$$

By scaling theses metrics suitably, namely, $\bar{g}_{i}=r_{h}\left(x_{i}\right)^{-2} g_{i}$, then 
1. $\bar{r}_{h}\left(x_{i}\right)=1$ and $\bar{r}_{h}(x)$ is bounded below on balls of finite distance to $x_{i}$, which follows from scale invariant property of Harmonic norm [Petersen97,

$$
\left\|x \in\left(M, \lambda^{2} g\right)\right\|_{C^{m, \alpha}, \lambda r}=\|x \in(M, g)\|_{C^{m, \alpha}, r}
$$

2. dist $_{\bar{g}_{i}}\left(x_{i}, \partial B_{i}\right) \rightarrow \infty$, since the ratio in (3.3) is scale invariant;

3. $\left|W^{+}\left(\bar{g}_{i}\right)\right|+\left|S\left(\bar{g}_{i}\right)\right| \leq r_{h}^{2}\left(x_{i}\right) \Lambda \rightarrow 0$, and the curvature have $\epsilon-$ small $L^{2}$ norm

$$
C_{S}\left\{\int_{B\left(x, \frac{2 r}{r_{h}\left(x_{i}\right)}\right)}\left|R m\left(\bar{g}_{i}\right)\right|^{2} d \bar{\nu}_{i}\right\}^{\frac{1}{2}} \leq \epsilon_{0},
$$

with respect to the metric $\bar{g}_{i}$.

We now consider, the sequence of pointed Riemannian manifolds

$$
\left\{\left(B_{i}\left(x_{i}, \frac{r}{r_{h}\left(x_{i}\right)}\right), x_{i}, \bar{g}_{i}\right) \subset\left(M_{i}, x_{i}, \bar{g}_{i}\right)\right\},
$$

by the fundamental theorem of convergence theory 3.3 , then the sequence subconvergent, in the pointed $C^{1, \beta}$ topology $(\forall \beta<\alpha)$, uniformly on compact subsets, to a complete $C^{1, \alpha}$ Riemannian manifold $(N, \bar{x}, h)$.

Claim 1. The convergence is actually better, namely in the $C^{1, \alpha}$ topology, where $\alpha$ is given by the hypothesis of the lemma.

Moreover, we can even prove more than we need, i.e. the convergence is in the $C^{1, \alpha} \cap W^{2, p}$ topology, for any $\alpha<1$ and $1<p<\infty$. By Soblev embedding theorem, $W^{2, p} \subset C^{1, \alpha}$ if $p>n$, so it suffices to prove the convergence is in the $W^{2, p}$ topology. To see this, by theorem 2.3, we know that the prescribed self - dual Weyl tensor and scalar curvature equation (2.2) is an elliptic system of partial differential equations of second order under harmonic coordinate:

$$
\left\{\begin{array}{l}
W^{+}=L\left(g^{-1} \partial \partial g\right)+Q_{1}(\partial g, \partial g) \in L^{\infty} \\
S(g)=-\frac{1}{2} g^{i j} g^{k l} \frac{\partial^{2}}{\partial x^{k} \partial x^{l}} g_{i j}+Q_{2}(\partial g, \partial g) \in L^{\infty}
\end{array}\right.
$$

where $L$ denotes linear combination, $Q$ is a quadratic term in the first order derivatives of $g$. For a priori estimate, since $\left\|g_{i j}-\delta_{i j}\right\|_{C^{1, \alpha}}<C$, if necessary we can make $C$ small, the above system actually can be viewed as a uniform linear elliptic system of $g_{i j}$ with $C^{1, \alpha}$ coefficients. By the a priori estimate (2.5), the $L^{p}$ theory for elliptic systems gives a uniform bound on $\|g\|_{W^{2, p}}$ for any $1<p<\infty$,

$$
\|g\|_{W^{2, p}} \leq C\left(\|g\|_{L^{p}}+\|Q(\partial g, \partial g)\|_{L^{p}}+\left\|W^{+}(g)\right\|_{L^{p}}+\mid S(g) \|_{L^{p}}\right) \leq C .
$$

As a consequence, the convergence is in the $C^{1, \alpha} \cap W^{2, p}$ topology, for any $\alpha<1$ and $1<p<\infty$.

More precisely, the $\left(B_{i}\left(\frac{r}{r_{h}\left(x_{i}\right)}\right), x_{i}, \bar{g}_{i}\right)$ are covered by harmonic coordinates that converge in the $C^{2, \alpha}$ topology to the harmonic coordinates on limit space $N$, and the metric coefficients $\bar{g}_{i}$ converge in the $C^{1, \alpha}$ topology to $h$. 
Since the $C^{1, \alpha}$ norm is continuous and harmonic radius is continuous with respect to $C^{1, \alpha}$ or $W^{2, p}$ convergence [Anderson90 Petersen97, and consequently,

$$
r_{h}(\bar{x})=1,\|\bar{x} \in(N, h)\|_{C^{1, \alpha}, r_{h}(\bar{x})} \geq \frac{C}{2}>0 .
$$

Claim 2. $h$ is a smooth Riemannian metric and $(N, h)$ is isometric to the Euclidean space $\left(\mathbb{R}^{4}, g_{E}\right)$.

Since the convergence is in the $C^{1, \alpha} \cap W^{2, p}$ topology, we can therefore conclude that the limit metric $h$ is a weak $C^{1, \alpha} \cap W^{2, p}$ solution of the elliptic system,

$$
\left\{\begin{array}{l}
W^{+}=L\left(g^{-1} \partial \partial g\right)+Q_{1}(\partial g, \partial g)=0 \\
S(g)=-\frac{1}{2} g^{i j} g^{k l} \frac{\partial^{2}}{\partial x^{k} \partial x^{l}} g_{i j}+Q_{2}(\partial g, \partial g)=0
\end{array}\right.
$$

namely, the anti self dual or Kähler metric with zero scalar curvature is a second order divergence form qusi-linear elliptic system of the metric modulo diffeomorphisms by theorem 2.3. With the a priori estimate (2.5) and a standard bootstrap argument, and also Sobolev embedding theorem, we conclude that the metric $h$ is actually a smooth (in fact, analytic) Riemannian metric with

$$
C_{S}\left\{\int_{N}|R m(h)|^{2} d \nu_{h}\right\}^{\frac{1}{2}} \leq \epsilon_{0} .
$$

If $\epsilon=\epsilon_{0}$ is sufficiently small (which will depends only on the Sobolev constant), by the $\epsilon$ - rigidity theorem 3.4 we conclude that $R m(h) \equiv 0$, i.e. $N$ is flat. On the other hand, bounded Sobolev constant implies Euclidean volume growth. Consequently, $(N, h)$ is isometric to the Euclidean space $\left(\mathbb{R}^{n}, g_{E}\right)$.

Since the Euclidean space admits global harmonic coordinates, i.e.

$$
r_{h}(x)=\infty, \quad\left\|x \in\left(\mathbb{R}^{n}, g_{E}\right)\right\|_{C^{1, \alpha}, r}=0, \forall r>0 .
$$

However, this violates (3.5).

Now we can prove the main theorem 1.1 which is an immediate consequence of the main lemma 3.5 on harmonic radius estimate and the fundamental theorem 3.3 of convergence theory.

Theorem 3.6. With the same hypothesis in theorem 1.1 then there exist a subsequence $\{j\} \subset\{i\}$ such that $\left(M, g_{j}\right)$ converge to a compact metric space $\left(M_{\infty}, g_{\infty}\right)$ in the $C^{1, \alpha}$ topology outside the finite singular set $S=\left\{x_{1}, \cdots, x_{s}\right\}$.

Proof. As in the case of bounded Ricci curvature or or Bach flat metric with constant scalar curvature, take $\epsilon=\epsilon_{0}$ in theorem 3.4, consider the sets

$$
\mathcal{R}_{i}(r)=\left\{x \in M_{i} \mid\left\{\int_{B(x, 2 r)}|R m|^{\frac{n}{2}}\right\}^{\frac{n}{2}}<\epsilon_{0}\right\}
$$

and

$$
\mathcal{S}_{i}(r)=\left\{x \in M_{i} \mid\left\{\int_{B(x, 2 r)}|R m|^{\frac{n}{2}}\right\}^{\frac{n}{2}} \geq \epsilon_{0}\right\}
$$


then $M_{i}=\mathcal{R}_{i}(r) \cup \mathcal{S}_{i}(r)$, and also $\mathcal{R}_{i}\left(r_{1}\right) \subset \mathcal{R}_{i}\left(r_{2}\right), \mathcal{S}_{i}\left(r_{1}\right) \supset \mathcal{S}_{i}\left(r_{2}\right)$, for any $r_{1}>r_{2}$.

For all $x \in \mathcal{R}_{i}(r)$, by the main lemma 3.5 we have the estimate on $C^{1, \alpha}$ harmonic radius,

$$
r_{h}(x) \geq \kappa_{0} r
$$

where $\kappa_{0}=C\left(C_{S}, \Lambda\right)$. On the other hand, the uniform Sobolev constant implies noncollapsing, namely, $\operatorname{Vol}(B(x, r)) \geq C\left(C_{S}\right) r^{n}$. Then the covering number (see definition 3.2) on any compact subset of $\mathcal{R}_{i}(r)$ on the scale $\inf _{x \in \mathcal{R}_{i}(r)} r_{h}(x) \geq$ $\kappa_{0} r$ can be bounded by

$$
\operatorname{Cov}\left(\frac{1}{10} \kappa_{0} r\right) \leq \frac{\operatorname{Vol}(M)}{\operatorname{Vol}\left(B\left(x, \frac{1}{10} \kappa_{0} r\right)\right)} \leq \frac{C\left(C_{S}, \Lambda\right)}{r^{4}} .
$$

With the fundamental convergence theorem 3.3 the sequence $\left(\mathcal{R}_{i}(r), g_{i}\right)$ is $C^{1, \alpha}$ subconvergent to a $C^{1, \alpha}$ (open) Riemannian manifold $\left(\mathcal{R}_{\infty}(r), g_{\infty}\right)$ on the compact set.

To construct the limit space, we will be brief since this step is quite standard, see for example Anderson89. BKN89. Tian90, and also Anderson05] TV05b].

We now choosing a sequence $\left\{r_{j}\right\} \rightarrow 0$ with $r_{j+1}<\frac{1}{2} r_{j}$, pepeat the above construction by choosing subsequence, we still denote $\{j\}$. Since $\mathcal{R}_{i}\left(r_{j}\right) \subset$ $\mathcal{R}_{i}\left(r_{j+1}\right)$, then we have a sequence of limit spaces with natural inclusions

$$
\mathcal{R}_{\infty}\left(r_{j}\right) \subset \mathcal{R}_{\infty}\left(r_{j+1}\right) \subset \cdots \subset \mathcal{R}_{\infty}:=\operatorname{dir} . \lim \mathcal{R}_{\infty}\left(r_{j}\right)
$$

By the $C^{1, \alpha}$ convergence, $\left(\mathcal{R}_{\infty}, g_{\infty}\right)$ is $C^{1, \alpha}$ (open) Riemannian manifold, and there are $C^{2, \alpha}$ smooth embedding $F_{i}:\left(\mathcal{R}_{\infty}, g_{\infty}\right) \rightarrow\left(M, g_{i}\right)$ such that $F_{i}^{*} g_{i} \rightarrow$ $g_{\infty}$ in the $C^{1, \alpha}$ topology on any compact set of $\mathcal{R}_{\infty}$.

Letting $\left\{B\left(x_{k}^{i}, \frac{r}{4}\right)\right\}_{k \in \mathbb{N}}, r<\frac{1}{4} \rho_{0}$, where $\rho_{0}$ is the Euclidean volume growth scale in theorem 4.1 be a collection of a maximal family of disjoint geodesic balls in $M_{i}$, then $M_{i} \subset \cup_{k} B\left(x_{k}^{i}, r\right)$. There is a uniform bound, independent of $i$, on the number of points $\left\{x_{k}^{i} \in S_{i}(r)\right\}$, which follows from

$$
m \leq \sum_{i=1}^{m} \epsilon_{0}^{-2} \int_{B\left(x_{k}^{i}, 2 r\right)}|R m|^{2} \leq C \epsilon_{0}^{-2} \int_{M_{i}}|R m|^{2}
$$

where $C=\sup _{x \in M_{i}} \frac{\operatorname{Vol}\left(B\left(x, \frac{9 r}{4}\right)\right.}{\operatorname{Vol}\left(B\left(x, \frac{r}{4}\right)\right.} \leq C\left(C_{S}, \Lambda\right)$. The last inequality holds because we have upper bound of the volume growth (4.1).

Let $\left\{r_{j}\right\}$ be as above without loss generality, we will assume $m$ is fixed, i.e. the number of mutually disjoint balls, which is centered in $S_{i}\left(r_{j}\right)$ and has radius $\frac{r_{j}}{4}$, is independent on $i$ and $j$. As a consequence, every point of $S_{i}\left(r_{j}\right)$ is contained in a ball of diameter no greater than $m r_{j}$. Hence, most of the volume $\left(M_{i}, g_{i}\right)$ is contained in $\mathcal{R}\left(r_{j}\right)$. Using the embedding $F_{i}^{j}:\left(\mathcal{R}_{\infty}\left(r_{j}\right), g_{\infty}\right) \rightarrow$ $\left(\mathcal{R}_{i}\left(r_{j}\right), g_{i}\right)$, we see that for any fixed $j$, and $i$ sufficiently large, arbitrarily large compact subsets od $R_{\infty} \backslash R_{\infty}\left(r_{j}\right)$ are almost isometrically embedded into $m$ disjoint balls of radius $r_{j}$. Letting $j \rightarrow \infty$, it follows that the the boundary components shrink to points with respect $g_{\infty}$. In other words, one can add 
finite points $\mathcal{S}_{\infty}=\left\{x_{1}, \cdots, x_{m}\right\}$ to $\mathcal{R}_{\infty}$ such that $M_{\infty}:=\mathcal{R}_{\infty} \cup \mathcal{S}_{\infty}$ is complete with respect to the length structure $g_{\infty}$, i.e. the Riemannian metric has a $C^{0}$ extension across the singularity. Moreover, $\left(M_{i}, g_{i}\right)$ sub convergent to $M_{\infty}$ in the Gromov - Hausdorff topology and the volume of geodesic ball (may contain singularity) is continuous with respect the $C^{1, \alpha}$ convergence (off finite singularity).

We now examine the topological structure near the singularity by essential studying the tangent cones at the singularity. Fix $p \in S_{\infty} \subset M_{\infty}$, let $r(x)=$ $\operatorname{dist}(x, p)$ and denote the annulus around $p$ to be

$$
A\left(r_{1}, r_{2}\right)=\left\{x \in M_{\infty} \mid r_{1}<r(x)<r_{2}\right\},
$$

where $r_{1}<r_{2}<\operatorname{dist}\left(p, S_{\infty} \backslash\{p\}\right)$. By the $C^{1, \alpha} \cap W^{2, p}$ convergence, recall the $C^{2, \alpha}$ smooth embedding $F_{i}:\left(\mathcal{R}_{\infty}, g_{\infty}\right) \rightarrow\left(M, g_{i}\right)$, the curvature will converge in the $L^{p}-$ sense, and then

$$
\int_{F_{i}\left(\mathcal{R}_{\infty}\right)}\left|R m\left(g_{i}\right)\right|^{2}<\infty, \forall i
$$

In particular, for $\epsilon_{0}$ in theorem 3.4 there is an $r_{0}>0$ such that

$$
\int_{F_{i}\left(A\left(0, r_{0}\right)\right)}\left|R m\left(g_{i}\right)\right|^{2} \leq \epsilon_{0}^{2}, \forall i \text {. }
$$

Now we do blow up analysis on $M_{\infty}$, it is equivalent to blow up the sequence. Namely, given any sequence $r_{j} \rightarrow 0$, let $j \rightarrow 0$, the metric annulus $\left(F_{i}\left(A\left(\frac{s_{j}}{j}, j s_{j}\right)\right), \frac{1}{s_{j}^{2}} g_{i}\right)$ (by taking diagonal sequence) sub - converge to $C^{1, \alpha} \cap W^{2, p}$ annulus $\left(A_{\infty}(0, \infty), g_{\infty}\right)$, where $g_{\infty}$ weak solution of anti self - dual with zero scalar curvature equation. With the regularity theory of Elliptic equation, it follows that $\left(A_{\infty}(0, \infty), g_{\infty}\right)$ is smooth. On the other hand, by (3.8), we know

$$
\int_{A(0, \infty)}\left|R m\left(g_{\infty}\right)\right|^{2} \leq \epsilon_{0}^{2}
$$

with Sobolev constant, we conclude that each component of $\left(A_{\infty}(0, \infty), g_{\infty}\right)$ is isometric to the Euclidean cone on a space form $S^{3} / \Gamma$ for some finite subgroup of $O(4)$.

If one has lower Ricci curvature, then the limit orbifold is irreducible, which is proved in Anderson89 by means of the Cheeger - Gromoll splitting theorem. In our case, there may be more than one cones associated to one singularity. If we perform a standard bubble analysis, one can estimate the precise bound on the end of associated ALE space, which in turn implies a bound on the number of cones at each singular point, depending only on $\left\|R i c_{-}\right\|_{L^{2}}, C_{S}$, see Carron98 and TV08. This also give a alternative way to show the limit orbifold is irreducible if one has lower Ricci curvature. For Kähler metric, only irreducible singular points can occur in limit, i.e. orbifold point, see more details in TV05b.

It follows that the neighborhoods of each singular points is homomorphic to finite cones on spherical spaces forms. 
Remark 3. For the proof of theorem 1.2, the argument is similar. In fact, it is much easier to estimate the harmonic radius as did in the main lemma 3.5

With Bianchi identity, the laplacian of Ricci curvature is related to the Bach tensor (Kähler) and Scalar curvature [TV05a, so we have a coupled system:

$$
\left\{\begin{aligned}
\Delta R i c & =2 B+\frac{1}{3} \operatorname{Hess} S+R m * R i c \\
\Delta g & =Q(\partial g, \partial g)-2 R i c
\end{aligned}\right.
$$

Under the $C^{3, \alpha}$ harmonic coordinates, we have the improved estimate

$$
\|g\|_{C^{3, \alpha^{\prime}}}<C, \forall 0<\alpha^{\prime}<1 \text {; and }\|g\|_{W^{4, p}}<C, \forall 1<p<\infty .
$$

Moreover, the blow up limit will be flat since $\epsilon$ - rigidity theorem 3.4 hold for Bach flat (Kähler) metric with zero Scalar curvature. The left argument is similar and will be omitted here.

\section{Volume Growth Near Singularity}

We have already seen that the volume growth plays a crucial role in understanding the structure near the singular set, see (3.7). By lack of the volume comparison, we must find alternative approach to bound the volume on a fixed scale, i.e. for some $\rho>0$, there exist $V_{1}>0$ such that $\operatorname{Vol}(B(p, r)) \leq V_{1} r^{n}, \forall r<\rho$. For Bach flat metric with constant Scalar curvature, Gang Tian and Jeff Viaclovsky concluded that the volume does bound on all scale, and the bound depends only on the Sobolev constant and $L^{2}$ norm of curvature TV05a TV05b TV08. In fact, if we check their paper carefully, we will find that the argument also holds in our case, where we work in $C^{1, \alpha}$ category in place of the $C^{\infty}$ category. The difficulty caused by the concentrate of curvature. If we do blow up analysis carefully, as did in Einstein case [Nakajima92, there will bubble out some non - flat ALE space (tree) which will satisfy stronger geometric conditions; and consequently, we can bound the volume growth.

Theorem 4.1. Let $\left(M^{4}, g\right)$ be a compact oriented four manifold with bounded self - dual Weyl tensor and Scalar curvature, i.e. $\left|W^{+}(g)\right|+|S(g)| \leq \Lambda$; bounded Sobolev constant $C_{S}$; and also finite $L^{2}$ - curvature, i.e. $\|R m\|_{L^{2}}<\Lambda_{1}$. For some $\rho_{0}>0$, there exists a constant $V_{1}>\omega_{4}$, depending only upon $\Lambda, \Lambda_{1}, C_{S}$ such that

$$
\operatorname{Vol}(B(x, r)) \leq V_{1} r^{4}
$$

for all $x \in M$ and $0<r<\rho_{0}$.

Proof. The theorem can be established by proceeding the same bubble procedure in TV08. For reader's convenience, we will copy down their argument with some slight modification to give a full argument in our case.

In the first place, if the curvature does concentrate too much, i.e. for $\rho>0$,

$$
\int_{B(x, 2 \rho)}|R m|^{2}<\epsilon_{0}^{2}
$$


the volume growth will be controlled. In fact, by lemma 3.5] the harmonic radius of $B(x, \rho)$ is bounded below, namely, there is uniform constant $r_{0}$, such that, for all $y \in B(x, \rho)$,

$$
r_{h}(y)>r_{0} \rho, \quad\|y \in(M, g)\|_{C^{1, \alpha}, r_{0} \rho}<C,
$$

and consequently,

$$
\operatorname{Vol}(B(x, r)) \leq e^{2 C} \omega_{4} r^{4}, \forall r \leq \rho .
$$

For any metric $(M, g)$, define the maximal volume ratio on the scale $\rho$ as

$$
\operatorname{MV}(g, \rho)=\max _{x \in M, 0<r<\rho} \frac{\operatorname{Vol}(B(x, r))}{r^{4}} .
$$

Note that for any compact smooth four Riemannian manifold $(M, g)$,

$$
\lim _{\rho \rightarrow 0} \operatorname{MV}(g, \rho)=\omega_{4},
$$

where $\omega_{4}$ is the volume ratio of the Euclidean metric on $\mathbb{R}^{4}$.

In this paper, we consider the maximal volume ratio on finite scale rather than on all scale in TV08. On the one hand, the local non - inflated volume is enough to shrink the singular set to point; On the other hand, one will see, with lacking of $\epsilon$ - regularity, we can not prove the Euclidean volume growth on the large scale by volume comparison.

If the theorem is not true, then for any a sequence $\rho_{j} \rightarrow 0$ with $\rho_{j+1}<$ $\frac{1}{2} \rho_{j}$, if we fix $j$, there exists a sequence of metrics $\left(M, g_{i, j}\right)$, which satisfies the hypothesis in the theorem, but $M V\left(g_{i, j}, \rho_{j}\right) \rightarrow \infty$. By passing to a diagonal subsequence, for any a sequence $\rho_{i} \rightarrow 0$ with $\rho_{i+1}<\frac{1}{2} \rho_{i}$, there exists a sequence of metrics $\left(M, g_{i}\right)$, which satisfies the hypothesis in the theorem, but

$$
\operatorname{MV}\left(g_{i}, \rho_{i}\right) \rightarrow \infty, \text { as } i \rightarrow \infty \text {. }
$$

For this sequence, we can exact a subsequence (which for simplicity we continue to denote by the index $i$ ) and $r_{i}<\rho_{i}$ such that

$$
2 e^{2 C}=\frac{\operatorname{Vol}\left(B\left(x_{i}, r_{i}\right)\right)}{r_{i}^{4}}=\max _{r \leq r_{i}} \frac{\operatorname{Vol}\left(B\left(x_{i}, r_{i}\right)\right)}{r^{4}},
$$

where $e^{2 C}$ comes from (4.2).

We furthermore assume $x_{i}$ is choose so that $r_{i}$ is minimal, that is, the smallest radius such that

$$
\operatorname{Vol}\left(B_{g_{i}}(x, r)\right) \leq 2 e^{2 C} r^{4}, \forall x \in M_{i} \text { and } r \leq r_{i} .
$$

Note that the inequality

$$
\int_{B\left(x_{i}, 2 r_{i}\right)}\left|R m\left(g_{i}\right)\right|^{2} \geq \epsilon_{0}^{2}
$$


must hold, each ball with lager volume growth (singularity) takes at least $\epsilon_{0}$ of $L^{2}$ - curvature. Otherwise, by the estimate (4.2), we would have

$$
\operatorname{Vol}\left(B_{g_{i}}\left(x_{i}, r_{i}\right)\right) \leq e^{2 C} r_{i}^{4}
$$

which violates the choice of $r_{i}$ in (4.4).

Now, we consider the resealed metric $\tilde{g}_{i}=r_{i}^{-2} g_{i}$, so that $B_{g_{i}}\left(x_{i}, r_{i}\right)=$ $B_{\tilde{g}_{i}}\left(x_{i}, 1\right)$. From the choice of $x_{i}$ and $r_{i}$, the rescaled metrics $\tilde{g}_{i}$ have bounded volume ratio, in all of unit size.

From the main theorem 1.1, their exist a subsequence converges on compact subsets to a complete length space $\left(M_{\infty}, g_{\infty}, x_{\infty}\right)$ in the $C^{1, \alpha}$ topology off finite many singularities, where $\left(M_{\infty}, g_{\infty}, x_{\infty}\right)$ is a multi-fold, $g_{\infty}$ is a smooth anti self - dual metric with zero Scalar curvature. Further, from theorem 3.4 for the multi- fold case, see proposition 4.3 and claim 4.4, p.14 in [TV08], there exists a constant $A_{1}$ such that

$$
\operatorname{Vol}\left(B_{g_{\infty}}\left(x_{\infty}, r\right)\right) \leq A_{1} r^{4}, \text { for all } r>0
$$

One have seen that if $r_{i} \rightarrow 0$, then the blow up limit will be a smooth multi - fold with critical metric, which is crucial to conclude (4.6). This is the main reason that we consider the maximal volume ratio on the finite scale.

We next return the (sub)sequence $\left(M, g_{i}\right)$ and exact another subsequence so that

$$
2600 A_{1}=\frac{\operatorname{Vol}\left(B\left(x_{i}^{\prime}, r_{i}\right)\right)}{r_{i}^{\prime 4}}=\max _{r \leq r_{i}^{\prime}} \frac{\operatorname{Vol}\left(B\left(x_{i}^{\prime}, r\right)\right)}{r^{4}} .
$$

Again, we assume that $x_{i}^{\prime}$ is chosen so that $r_{i}^{\prime}$ is minimal, that is, the smallest radius for which

$$
\operatorname{Vol}\left(B_{g_{i}}(x, r)\right) \leq 2600 A_{1} r^{4}, \forall x \in M_{i} \text { and } r \leq r_{i}^{\prime}
$$

Clearly, $r_{i}<r_{i}^{\prime}<\rho_{i} \rightarrow 0$.

Arguing as above, we repeat the rescaled limit construction, but now with scaled metric $g_{i}^{\prime}=r_{i}^{\prime-2} g_{i}$, and basepoint $x_{i}^{\prime}$. We find a limiting multi - fold $\left(M_{\infty}^{\prime}, g_{\infty}^{\prime}, x_{\infty}^{\prime}\right)$, and a constant $A_{2} \geq 2600 A_{1}$ so that

$$
\operatorname{Vol}\left(B_{g_{\infty}^{\prime}}\left(x_{\infty}^{\prime}, r\right)\right) \leq A_{2} r^{4}, \text { for all } r>0
$$

For the same reason as in (4.5), we must have

$$
\int_{B_{g_{i}}\left(x_{i}, 2 r_{i}^{\prime}\right)}\left|R m\left(g_{i}\right)\right|^{2} \geq \epsilon_{0}^{2}
$$

Since the $L^{2}$ - curvature is finite, and each lager volume growth ball (singularity) takes at least $\epsilon_{0}$ of $L^{2}$ - curvature, it is reasonable to hope that the bubbling process will ended in finite step. But we need to be a little careful, as in Einstein case Nakajima92, there could be some overlap if any singular point lies in a ball centered at other singular point. 
So we next consider the ration $\frac{r_{i}^{\prime}}{r_{i}}$.

Case(i): there exists a subsequence(which we continue to index with $i$ ) satisfying $r_{i}^{\prime}<C r_{i}$ for some constant $C$.

Case(ii):

$$
\lim _{i \rightarrow \infty} \frac{r_{i}^{\prime}}{r_{i}}=\infty
$$

In Case(i) we proceed as follows: We claim that for $i$ sufficiently large, the balls $B\left(x_{i}, 2 r_{i}\right)$ (from the first subsequence) and $B\left(x_{i}^{\prime}, 2 r_{i}^{\prime}\right)$ (from the second) must disjoint because of choice in (4.7). To see this, if $B\left(x_{i}, 2 r_{i}\right) \cap B\left(x_{i}^{\prime}, 2 r_{i}^{\prime}\right) \neq \emptyset$, then $B\left(x_{i}^{\prime}, 2 r_{i}^{\prime}\right) \subset B\left(x_{i}, 6 r_{i}^{\prime}\right)$. Then (4.6) and (4.7) implies that

$$
\begin{aligned}
2600 A_{1}\left(r_{i}^{\prime}\right)^{4} & =\operatorname{Vol}\left(B\left(x_{i}^{\prime}, r_{i}^{\prime}\right)\right) \\
& <\operatorname{Vol}\left(B\left(x_{i}^{\prime}, 2 r_{i}^{\prime}\right)\right)<\operatorname{Vol}\left(B\left(x_{i}, 6 r_{i}^{\prime}\right)\right) \\
& \leq 2 A_{1}\left(6\left(r_{i}^{\prime}\right)^{4}\right)=2592\left(r_{i}\right)^{4}
\end{aligned}
$$

which is a contradiction (note the last inequality is true for $i$ sufficiently large since the volume is continuous under $C^{1, \alpha}$ topology (even with finite singularity), which is valid only in the Case (i)).

In case (ii), if the balls $B\left(x_{i}, 2 r_{i}\right)$ (from the first subsequence) and $B\left(x_{i}^{\prime}, 2 r_{i}^{\prime}\right)$ (from the second) are disjoint for all $i$ sufficiently large, then it will account at leat $2 \epsilon_{0}$ of $L^{2}$ - curvature. Otherwise, we look again at the scaling so that $r_{i}^{\prime}=1: \tilde{g}_{i}=\left(r_{i}^{\prime}\right)^{-2} g_{i}$, and basepoint $x_{i}^{\prime}$. Then in this rescaled metric,

$$
\operatorname{Vol}\left(B\left(x_{i}^{\prime}, 1\right)\right)=2600 A_{1} .
$$

As above, we have a limiting smooth multi - fold $\left(M_{\infty}^{\prime}, g_{\infty}^{\prime}, x_{\infty}^{\prime}\right)$, satisfying

$$
\operatorname{Vol}\left(B\left(x_{\infty}^{\prime}, 1\right)\right)=2600 A_{1}
$$

Since the metric is anti - self - dual with constant curvature, by the choice of $A_{1}$, we concluded that

$$
\int_{B_{g_{\infty}^{\prime}}\left(x_{\infty}, 2\right)}|R m|^{2}>\epsilon_{0}^{2} .
$$

There is now a singular point of convergence corresponding to the balls $B\left(x_{i}, r_{i}\right)$ in the first subsequence. But since we are in Case (ii) with $\lim _{i \rightarrow \infty} \frac{r_{i}^{\prime}}{r_{i}}=\infty$, in the $g_{i}^{\prime}$ metric, these balls must limit a point in $M_{\infty}^{\prime}$. The only possibility is that the original sequence satisfies

$$
\int_{B_{g_{i}}\left(x_{i}^{\prime}, 2 r_{i}^{\prime}\right)}\left|R m\left(g_{i}\right)\right|^{2}>2 \epsilon_{0}^{2},
$$

for all $i$ sufficiently large.

We repeat the above procedure, considering possible Cases (i) and (ii) at each step. At the $k$ th step, we can always account for at least $k \epsilon_{0}$ of $L^{2}-$ curvature. The process must terminate in finitely many steps from the bound $\left\|R m\left(g_{i}\right)\right\|_{L^{2}}<\Lambda_{1}$. This contradicts (4.3), which finishes the proof. 
We note that, it may happen that $\left(M_{\infty}, g_{\infty}\right)$ is a smooth Riemannian manifold, but the convergence is not in the $C^{1, \alpha}$ topology. In fact, the curvature concentrate part, corresponding to some nontrivial 2 - cycles in $M$, maybe shrink off in the limit. The topology 'decrease' and the singularity take away a certain quantity of energy of curvature.

Proposition 4.2. Let $\left(M, g_{i}\right)$ satisfies hypothesis in theorem 1.1, then

$$
\lim _{i \rightarrow \infty} \int_{M}\left|R m\left(g_{i}\right)\right| \geq \int_{\mathcal{R} \subset M_{\infty}}\left|R m\left(g_{\infty}\right)\right|^{2},
$$

with inequality if only if $M_{\infty}$ is $C^{1, \alpha}$ manifold diffeomorphic to $M$, and the convergence is in the $C^{1, \alpha}$ Cheeger - Gromov topology.

Proof. It is a straightforward consequence of the Bubble analysis. Since the converge is taking in the $C^{1, \alpha} \cap W^{2, p}$, then measure $\left|R m\left(g_{i}\right)\right|^{2} d v_{g_{j}}$ converge to

$$
\left|R m\left(g_{\infty}\right)\right| d v_{g_{\infty}}+\sum_{x_{i} \in \mathcal{S}_{\infty}} a_{i} \delta_{x_{i}}
$$

in the $L^{p}$ sense, where $\delta_{x_{i}}$ is the Dirac measure supported at $x_{1}$, and $a_{k}$ is given by

$$
a_{k}=\sum_{\left(N_{k}^{*}, h_{k}^{*}\right)} \int_{\left(N_{k}^{*}, h_{k}^{*}\right)}\left|R m\left(h_{k}^{*}\right)\right|^{2}
$$

Here $\left(N_{k}^{*}, h_{k}^{*}\right)$ is the bubble tree associated to the singular point $x_{k}$, see clear description of bubble tree in Nakajima92. The equality implies there are no curvature concentration occurred and thus no singularities in the limit.

Question 1. Does the maximum of sectional curvature (curvature singularity) always occurred in the singular part $\mathcal{S}_{i}(r)$ in theorem 3.6.

Since there is no $\epsilon$ - regularity theorem, in appearance, curvature may blow up even if small energy. If this phenomena does not occur, combined with the results and methods in Anderson89, one may show that under certain circumstances, in fact orbifold singularities do not arise in the limit.

For instance, in dimension 4 , one can introduce the bound

$$
\inf \left\{\operatorname{area}(\Sigma):[\Sigma] \neq 0 \in H_{2}(M, \mathbb{Z}) / \text { torsion } \geq a>0 .\right\}
$$

Then the deepest bubble $N$ associated to each singularity will be a non flat ALE space with $\operatorname{dim} H_{2}(N) \neq 0$. Then there exists $\left[\Sigma_{i}\right] \neq 0$ in $H_{2}\left(M_{i} ; \mathbb{Z}\right)$ with area Area $\left(\Sigma_{i}\right) \rightarrow 0$ as $i \rightarrow \infty$, i.e. the metric to become degenerate along $\left[\Sigma_{i}\right]$, which contradict to (4.8). Consequently, the convergence of Riemannian manifolds in theorem 1.1 will be in the $C^{1, \alpha}$ topology.

\section{References}

[Anderson89] M. Anderson: Ricci curvature bounds and Einstein metrics on compact manifolds. J. Amer. Math. Soc. 2 (1989), 455 - 490. 
[Anderson90] M. Anderson: Convergence and rigidity of manifolds under Ricci curvature bounds. Invent. Math.102 (1990), 429 - 445.

[AnCh92] M. Anderson, J. Cheeger: $C^{\alpha}$ compactness for manifolds with Ricci curvature and injectivity radius bounded below. J. Differ. Geom. 35 (1992), $265-281$.

[Anderson05] M. Anderson: Orbifold Compactness for Spaces of Riemannian Metrics and Applications. Math. Ann. 331, no 4 (2005), 739 - 778.

[BKN89] S. Bando, A. Kasue, H. Nakajima: On the construction of coordinates at infinity on manifolds with fast curvature decay and maximal volume growth. Invent. math. 97 (1989), 313 - 349.

[Besse87] Arthur L. Besse: Einstein Manifolds. Reprint of the 1987 edition. Classics in Mathematics. Springer-Verlag, Berlin, 2008.

[Calabi82] E. Calabi, Extremal Kähler metric. Seminar on Differential Geometry, 259 - 290, Ann. of Math. Stud., 102, Princeton Univ. Press, Princeton, N.J., 1982.

[Carron98] Gilles Carron: Une suite exacte en $L^{2}$ - cohomologie. Duke Math. J. 95 (1998), no. 2, $343-372$.

[Carron10] G.Carron: Some old and new result about rigidity of critical metric. arXiv: 1012.0685 [math.DG].

[CCT02] J.Cheeger, T.H. Colding, G.Tian: On the singularities of spaces with bounded Ricci curvature. GAFA, Geom. funct. anal. Vol. 12 (2002), 873 914.

[ChHe08] X.X.Chen, W.Y.He: On the Calabi Flow. J. Amer. Math. Soc. 2 (2008), 539-570.

[DK90] S.K.Donaldson, P.B.Kronheimer: The Geometry of Four Manifolds. Oxford Science Publications. The Clarendon Press, Oxford University Press, New York, 1990.

[GW88] R. Greene, H. Wu: Lipschitz convergence of Riemannian manifolds. Pac. J.Math.131(1988), 119 -141.

[Jost84] J. Jost: Harmonic mappings between Riemannian manifolds. Proceedings of the Centre for Mathematical Analysis, Australian National University, 4. Australian National University, Centre for Mathematical Analysis, Canberra, 1984.

[KK92] A.D.King, D.Kotschick: The deformation theory of anti - self - dual conformal structures. Math. Ann. 294 (1992), 591 - 609. 
[KT87] R. Kobayashi, A. Todorov: Polarized period map for genaralized K3 surfaces and the moduli of Einstein metrics. Têhoku Math. Journ. 39 (1987), $341-363$.

[Morrey66] C.B.Morrey: Multiple integrals in the calculus of variations. Reprint of the 1966 edition. Classics in Mathematics. Springer-Verlag, Berlin, 2008.

[Nakajima92] H. Nakajima: Convergence theorem of Einstein metrics and ALE spaces. Sugaku 44 (1992), no 2, 346 - 372.

[Palais68] R. S. Plasis: Foundations of global analysis. W.A. Benjamin, New York, 1968.

[Petersen97] P. Petersen: Convergence theorems in Riemannian geometry. In Comparison geometry (Berkeley, CA, 1993C94), volume 30 of Math. Sci. Res. Inst. Publ., pages 167C202. Cambridge Univ. Press, Cambridge, 1997.

[Schoen87] R.Schoen: Variational Theory for the Total Scalar Curvature Functional for Riemannian Metrics and Related Topics. Topics in calculus of variations (Montecatini Terme, 1987), 120C154, Lecture Notes in Math., 1365, Springer, Berlin, 1989.

[Tian90] Gang Tian: On Calabi conjecture for complex surfaces with positive first Chern class. Invent. Math. 101 (1990), no. 1, 101 -172.

[Tian08] Gang Tian: Aspects of Metric geometry of four Manifolds. www.crm.cat/Publications/quaderns/Quadern48.2_tian2.pdf.

[TV05a] Gang Tian, Jeff. Viaclovsky: Bach - flat asymptotically locally Euclidean metrics. Invent. Math. Vol. 160 (2005), No. 2, 357 - 415.

[TV05b] Gang Tian, Jeff. Viaclovsky: Moduli spaces of critical Riemannian metrics in dimension four. Advances in Math. Vol. 196 (2005), No. 2, 346 -372 .

[TV08] Gang Tian, Jeff. Viaclovsky: Volume growth, curvature decay, and critical metrics. Comment. Math. Helv. 83 (2008), no. 4, 889 - 911.

Yiyan Xu, School of Mathematical Sciences, Peking University, BeiJing, China, 100871 xuyiyan@math.pku.edu.cn 